\title{
Time-resolved imaging of the laser forward transfer of liquids
}

\author{
M. Duocastella, J. M. Fernández-Pradas, J. L. Morenza, and P. Serra ${ }^{\text {a) }}$ \\ Departament de Física Aplicada i Òptica, Universitat de Barcelona, Martí i Franquès 1, \\ E-08028 Barcelona, Spain
}

(Received 20 March 2009; accepted 22 September 2009; published online 29 October 2009)

\begin{abstract}
Time-resolved imaging is carried out to study the dynamics of the laser-induced forward transfer of an aqueous solution at different laser fluences. The transfer mechanisms are elucidated, and directly correlated with the material deposited at the analyzed irradiation conditions. It is found that there exists a fluence range in which regular and well-defined droplets are deposited. In this case, laser pulse energy absorption results in the formation of a plasma, which expansion originates a cavitation bubble in the liquid. After the further expansion and collapse of the bubble, a long and uniform jet is developed, which advances at a constant velocity until it reaches the receptor substrate. On the other hand, for lower fluences no material is deposited. In this case, although a jet can be also generated, it recoils before reaching the substrate. For higher fluences, splashing is observed on the receptor substrate due to the bursting of the cavitation bubble. Finally, a discussion of the possible mechanisms which lead to such singular dynamics is also provided. (C) 2009 American Institute of Physics. [doi:10.1063/1.3248304]
\end{abstract}

\section{INTRODUCTION}

The possibility of printing tiny amounts of functional materials in a controlled and precise way is a great challenge in diverse areas, such as in the manufacturing of organic electronics, where this technology offers great flexibility and potential for low production costs. ${ }^{1}$ It could also set a new era in tissue engineering applications, allowing the construction of artificial organs combining the deposition of cells and biomaterials, in the so called organ printing. ${ }^{2}$ Moreover, among others, it could allow the fabrication of miniaturized devices in areas such as biosensing and biochemical analysis, in which miniaturization presents advantages such as lab-ona-chip integration, multianalyte detection, and minimization of sample volumes. ${ }^{3}$

In all the previous cases, and especially in those in which biological elements come into play, the demanded printing technologies must assure the functionality of the transferred specimens, and should try to minimize the difficulties that may appear during the printing process, such as contamination. Therefore, fast techniques which avoid direct mechanical contact with the deposited material would be desirable. Noncontact direct writing techniques meet these requirements, and among them ink-jet printing ${ }^{4,5}$ is the most extended one. However, ink-jet printing is seriously limited by the presence of a nozzle, which can be clogged by dried ink; in addition, only liquids with a limited range of rheological properties can be printed through this technique. ${ }^{4}$

An alternative to ink-jet printing is laser-induced forward transfer (LIFT), which avoids the constraints of a nozzle, while it fulfills the mentioned requirements. ${ }^{6}$ In the LIFT of liquids, laser pulses are used to transfer portions of material from a liquid donor film to a receptor substrate. The laser pulses are focused on the donor film through its supporting substrate, transparent to the laser radiation, impelling

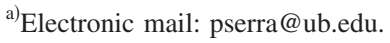

an unaffected fraction of the donor material toward the receptor. Since many solutions of interest are transparent to the laser radiation, an absorbing layer is usually placed between the donor film and its support. ${ }^{7,8}$ The translation of both donor and receptor with respect to the laser beam allows printing micropatterns of any predefined two-dimensional geometry. The technique has also a high degree of spatial resolution: under appropriate irradiation conditions the material can be deposited in the form of single microdroplets, ${ }^{9}$ which can have diameters as small as a few microns. ${ }^{10}$ Moreover, LIFT can be used for printing different complex materials, such as inorganic inks or pastes, ${ }^{11}$ organic polymers, ${ }^{12}$ and even biological solutions. ${ }^{8,13-15}$ The feasibility of the technique for depositing these materials has been proved through the fabrication of diverse functional devices such as microbatteries, ${ }^{11}$ solar cells, ${ }^{16}$ organic light-emitting diodes, ${ }^{17}$ or biosensors. ${ }^{18,19}$

Such interesting features prompted several studies on the transfer process which takes place during the LIFT of liquids. These studies ${ }^{9,10,14,15,20}$ were mainly focused on the analysis of the effects that different technological parameters have on the morphology of the transferred material. This allowed improving the performance of the technique, although it also revealed the need for the elucidation of the transfer mechanisms in order to set the basis for parameters optimization. In a first attempt to unveil such mechanisms, a time-resolved study of the LIFT of a viscous nanopowder suspension was carried out. ${ }^{21}$ In that work, a study of the process dynamics at different laser fluences was reported; however, for such a suspension and irradiation conditions, material deposition did not take place in the form of a single droplet on the receptor, but rather as a set of multiple droplets. The time-resolved images of the LIFT process for a biomolecule containing solution ${ }^{22}$ did not either provide the information necessary to unveil the transfer mechanism. In that case, a direct correlation between time-resolved images and deposited material could not be carried out, since the 
conditions at which images were acquired differed from those of the deposits. Recently, and also through timeresolved imaging, we demonstrated that liquid transfer proceeded in a different way when deposition was carried out under conditions really leading to the formation of single circular microdroplets. ${ }^{23}$ In that case liquid ejection occurred in the form of long, uniform and regular jets which propagated from the film to the receptor substrate. In the present work, we use time-resolved imaging to undertake a more complete and detailed study of the transfer dynamics through the analysis of the different possible liquid ejection mechanisms which take place at different laser fluences, and to investigate the correlation between these mechanisms and the morphology of the deposited material. Finally, we discuss on the possible causes which lead to the observed singular dynamics.

\section{EXPERIMENTAL}

The LIFT experiments were performed using a setup ${ }^{23}$ which consists of a pulsed Nd:YAG (yttrium aluminum garnet) laser (355 nm wavelength, $10 \mathrm{~ns}$ pulse duration, $1 \mathrm{~Hz}$ repetition rate), an optical system to guide and focus the radiation on the donor substrate, and a computer-controlled $x y z$ translation stage to allow the displacement of both donor and receptor substrates with respect to the laser beam. The focusing element is a microscope objective with a magnifying power of $15 \times$ and a numerical aperture of 0.32 .

The liquid solution employed in the current experiments was a model solution used in previous studies, ${ }^{9,23}$ which consists of a mixture of water and glycerol, both at a concentration of $50 \%(\mathrm{v} / \mathrm{v})$, plus the surfactant sodium dodecyl sulfate dissolved at a concentration of $2.0 \mathrm{mg} / \mathrm{ml}$. The surface tension of this solution was of about $35 \mathrm{mN} / \mathrm{m}$. A volume of $20 \mu \mathrm{l}$ of this solution was spread using a blade coater on top of a microscope glass slide coated with an absorbing layer of $\mathrm{Ti}(50 \mathrm{~nm}$ thick). This allowed obtaining a liquid film with a thickness of around $20 \mu \mathrm{m}$ (estimated through the weight measurement).

The liquid film was then placed parallel to a commercial poly-L-lysine coated glass slide, which acted as receptor substrate. The separation between the film and the substrate was $300 \mu \mathrm{m}$, kept using spacers. The whole system was then placed on the translation stages, in a position in which the Gaussian laser beam presented a diameter of $8 \mu \mathrm{m}$ on the Ti coating (full width at $1 / e^{2}$ maximum). Laser firing and translation of the stages were synchronized using an in-house computer program, so that a single laser pulse was fired at each position. This allowed obtaining a microarray of droplets separated $150 \mu \mathrm{m}$ apart, in which the laser pulse energy (and thus the laser fluence) was varied from row to row. The receptor substrate was then removed from the system in order to characterize the deposited droplets through optical microscopy and optical profilometry. The latter allowed obtaining the three-dimensional profiles of the droplets from which their volumes were calculated.

After receptor substrate removal (while preserving the donor substrate in its original location) high-speed images of the LIFT process from the side view were acquired in the

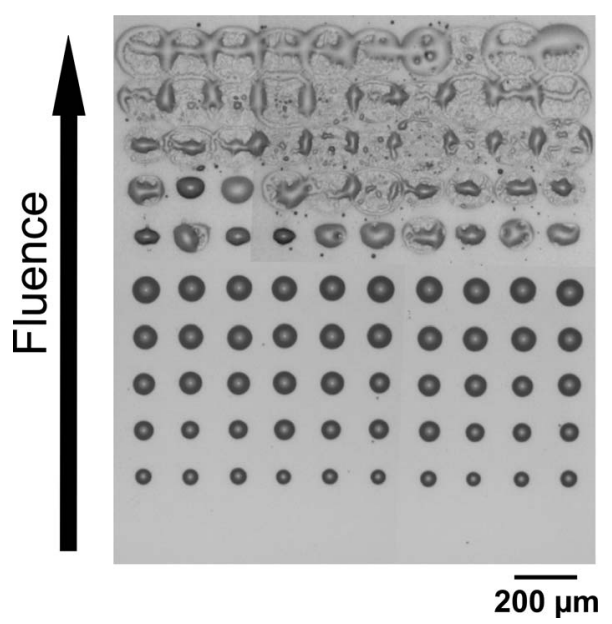

FIG. 1. Optical microscopy image of the microarray obtained at different laser fluences from 1 to $12 \mathrm{~J} / \mathrm{cm}^{2}$

fluence range used in the deposition experiment. The imaging system consisted of an illuminating source (a cw Nd:YAG laser, $532 \mathrm{~nm}$ wavelength, $20 \mathrm{~mW}$ power), a divergent lens (1 $\mathrm{m}$ focal length) to improve the uniformity of the illuminated region, a $40 \times$ magnification system composed of a microscope objective plus a teleobjective and, finally, a gated charged-coupled device (CCD) camera. All these elements were aligned and set up at grazing incidence respect to the donor surface, as described in Ref. 23. The resolution of the CCD camera was $288 \times 385$ pixels, with a 8 bits dynamic range, and it was intensified by a microchannel plate (MCP) which aperture was controlled by means of a pulse generator triggered by the laser pulse. A single image frame was acquired for each laser pulse, using an integration time of $100 \mathrm{~ns}$. Varying the time delay between the laser pulse and the MCP aperture, stop-action movies of the transfer process were reconstructed.

An additional experiment was carried out in which highspeed images of the process were acquired from the top view. In this occasion, a different configuration of the imaging system was used: the CCD camera was set up on top of the microscope objective that focuses the laser pulses on the donor film. In this way, this objective was also serving as a magnifying element for the acquisition of the time-resolved images. The experiment was performed without removing the receptor substrate, so that the droplets deposited on the receptor could be visualized through the CCD camera. This was achieved by using a continuous white lamp (150 W power) as illuminating source, which was situated below the receptor. This high power lamp provided enough light to visualize the printing process through the Ti coating despite its high reflectivity. The Ti coating was thin enough to allow a small fraction of the incoming light passing through the coating and being collected by the CCD detector.

\section{RESULTS}

\section{A. Microarray characterization}

An optical microscopy image of the deposited microarray is presented in Fig. 1. As it has already been reported in previous works, ${ }^{9,12,15,18}$ there exists a fluence range in which 


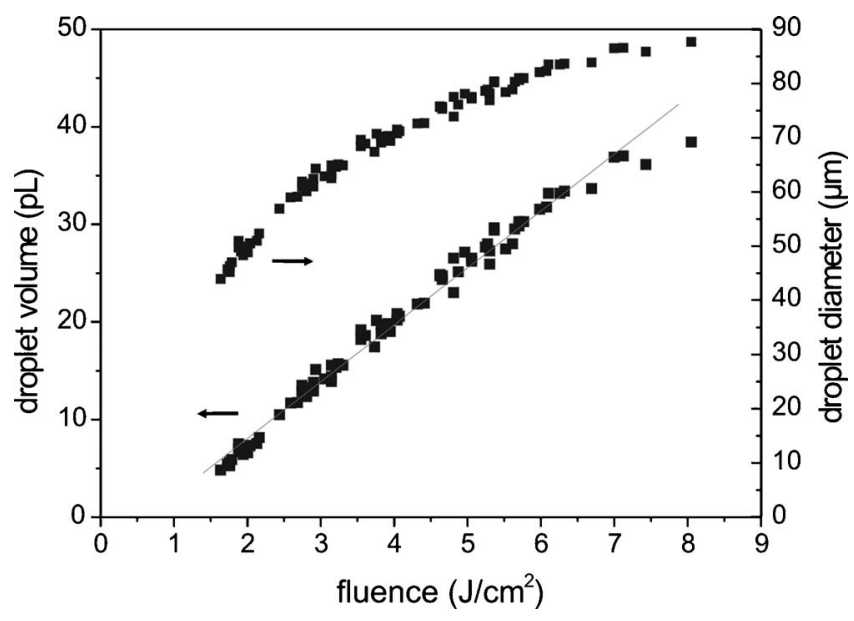

FIG. 2. Plots of the droplet volume and the droplet diameter vs the laser fluence

circular and well-defined droplets are obtained. However, at fluences out of this range different situations occur: at high fluences irregular droplets are deposited, and at even higher fluences only splashing is observed; on the other hand, at low fluences no material is transferred. Therefore, the conditions of technological interest, in which a controlled and precise deposition of liquids is required, correspond to an intermediate range of fluences between 1.5 and about $7.0 \mathrm{~J} / \mathrm{cm}^{2}$. In this range, the droplet dimensions increase as the laser fluence increases. The plot of the droplet volume versus the laser fluence (Fig. 2) presents an upward trend with a clear linear increase, as it has also been observed in prior works. ${ }^{9,15,20}$ The droplet diameter, which sets the resolution of the technique, plotted versus the laser fluence (Fig. 2) also presents an upward trend, but the increase is not linear. This contrasts with the behavior observed in the case of LIFT with thick absorbing polymeric layers, where a linear dependence was found.$^{14}$ In that case, however, microscopy images of the absorbing layer after transfer reveal plastic deformation of the polymer, instead of ablation. This indicates that the transfer mechanism would be probably different from the case of metallic absorbing layers, which can account for the difference observed in the relation between diameter and laser fluence.

\section{B. Time-resolved imaging}

In order to understand the transfer mechanisms which lead to the differences observed in the deposited material, four series of side view images of the transfer process were acquired using an ultrafast camera (Fig. 3). Each of these series corresponds to a different laser fluence. The four fluences were chosen to be representative of the different deposition situations that could occur: $1.2 \mathrm{~J} / \mathrm{cm}^{2}$ [Fig. 3(a)], in which no material is deposited; $2.4 \mathrm{~J} / \mathrm{cm}^{2}$ [Fig. 3(b)] and $5.6 \mathrm{~J} / \mathrm{cm}^{2}$ [Fig. 3(c)], in which well defined droplets are obtained; and finally $10 \mathrm{~J} / \mathrm{cm}^{2}$ [Fig. 3(d)], in which irregular droplets with splashing appear.

In all the frames of Fig. 3, the laser pulse was incident from above. The background is composed of two clearly differentiated regions, each one with a different gray tonality.
The upper dark gray part corresponds to the donor substrate, as the CCD was slightly tilted with respect to the zero grazing angle, and the brightest lower part corresponds to the free space. Moreover, two different fronts evolving into opposite directions can be distinguished. The upward front is the reflection of the downward one due to the presence of the $\mathrm{Ti}$ coating, which acts as a mirror. Therefore, only the downward front should be considered in the analysis.

In Fig. 3(a), corresponding to the lowest laser fluence, a protrusion is initially generated, which expands during the first $1.4 \mu \mathrm{s}$. Then, the protrusion starts suffering a gradual collapse from its sides while the central tip maintains its progression, leading to the formation of a small jet. At $4.1 \mu$ s the jet is fully developed, but it does not keep on growing. In fact, in the following frames it can be observed how the jet moves back to the liquid film, noting that recoil is much slower than expansion. Finally, at $100 \mu$ s the jet has become a very small protrusion which continues relaxing over a long time (images not shown).

At the fluences in which well-defined droplets are obtained, liquid transfer follows a quite singular dynamics. In Figs. 3(b) and 3(c), the initial expansion process followed by protrusion collapse and further jet formation undergoes an evolution similar to that shown in Fig. 3(a); even the time at which the jet is fully developed is similar for the three series of images (around $4 \mu \mathrm{s}$ ). Yet, it is interesting to notice that this initial expansion process is much more significant as the laser fluence increases, that is, the maximum dimensions of the protrusion increase with fluence. In fact, the balloonlike aspect acquired by the protrusion at the highest fluence [Fig. $3(c)$ ] clearly reveals the presence of a vapor bubble, which pushes the liquid away the film. After bubble collapse and once the jet is formed, however, the differences between the dynamics presented in Fig. 3(a), and those in Figs. 3(b) and 3(c) become relevant: in the latter two cases, once the jet is fully formed it keeps on advancing while maintaining its needlelike shape during the whole progression; the jet thickness decreases as its length increases, leading the jet to achieve a very high aspect-ratio. After the advancing front surpasses the field of view of the camera, it can only be appreciated that thinning proceeds, until the jet finally breaks into multiple parts. The breakup time depends on laser fluence: it occurs between 100 and $120 \mu \mathrm{s}$ at $2.4 \mathrm{~J} / \mathrm{cm}^{2}$ [Fig. 3(b)], while at $5.6 \mathrm{~J} / \mathrm{cm}^{2}$ [Fig. 3(c)] it takes place at around $50 \mu \mathrm{s}$. After the jet breakup, only a protrusion on the liquid film remains, which persists over several milliseconds (images not shown).

Concerning the transfer dynamics at higher fluences, a situation different from the previously described ones occurs [Fig. 3(d)]. In this case, a protrusion is also formed, which expands during the first hundreds of nanoseconds in a similar way as described above. However, at about 780 ns the protrusion tip becomes blurred, indicating bubble burst. At 980 ns, burst is evident, and in the following frames the evolution of the broken bubble reveals that it collapses from the sides, reducing its size, with evidence of turbulent flow in the central part. Finally, at $2.2 \mu$ s the bubble seems to be almost completely disintegrated. 
a)

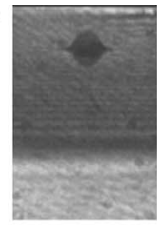

380ns

b)

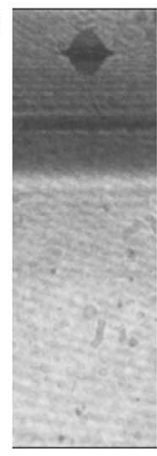

380ns

c)

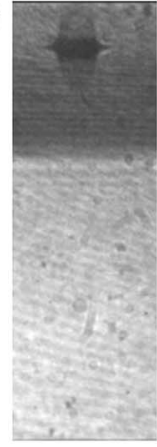

180ns

d)

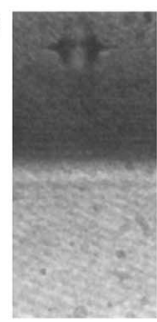

80ns

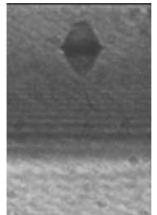

980ns

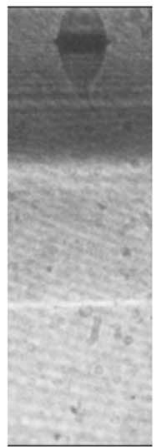

$1.4 \mu \mathrm{s}$

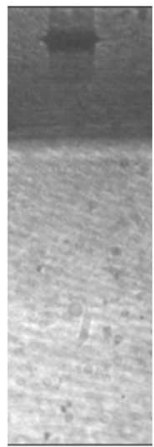

$380 \mathrm{~ns}$

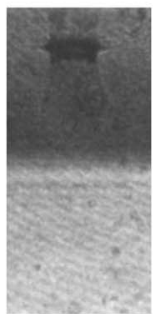

180ns

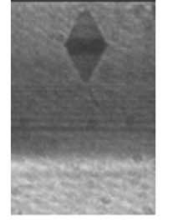

$1.4 \mu \mathrm{s}$

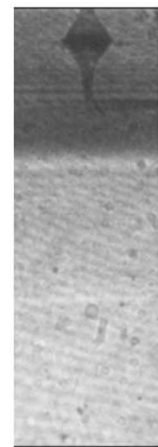

$1.8 \mu \mathrm{s}$

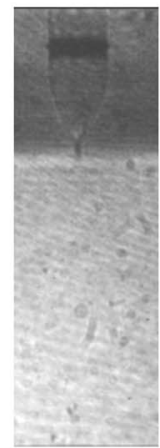

$1.2 \mu \mathrm{s}$

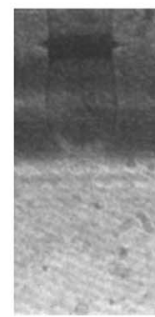

$580 \mathrm{~ns}$

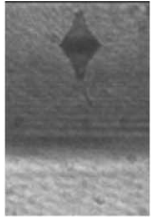

$1.8 \mu \mathrm{s}$

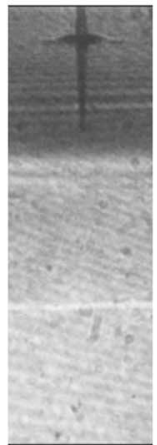

$4.1 \mu \mathrm{s}$

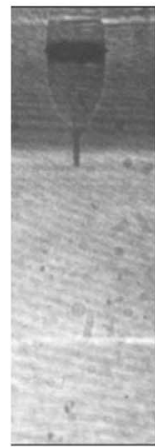

$1.4 \mu \mathrm{s}$

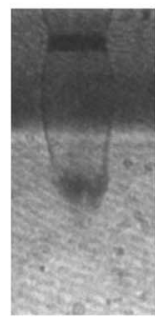

$780 \mathrm{~ns}$

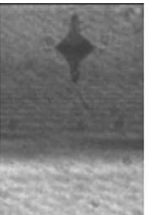

$2.2 \mu \mathrm{s}$

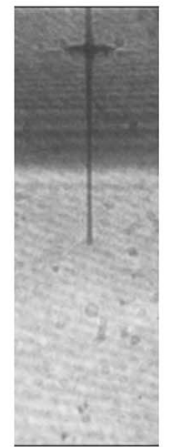

$10 \mu \mathrm{s}$

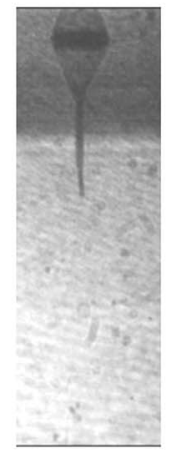

$1.8 \mu \mathrm{s}$

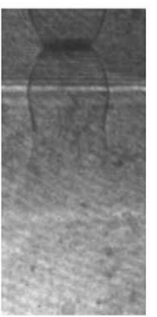

$980 \mathrm{~ns}$

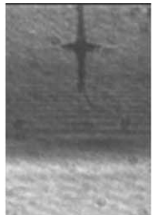

$4.1 \mu \mathrm{s}$

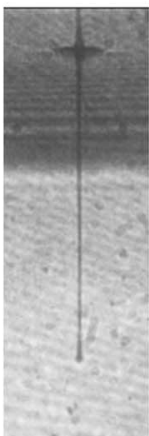

$20 \mu \mathrm{s}$

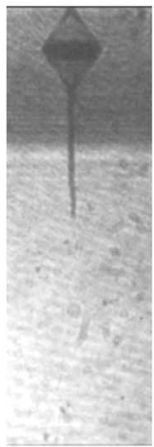

$2.2 \mu \mathrm{s}$

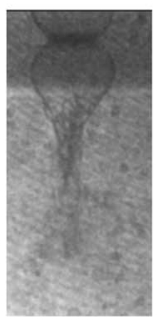

$1.4 \mu \mathrm{s}$

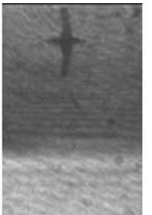

$15 \mu \mathrm{s}$

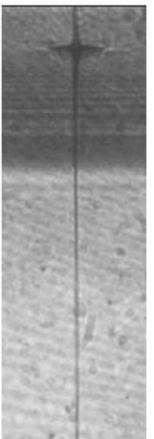

$30 \mu \mathrm{s}$

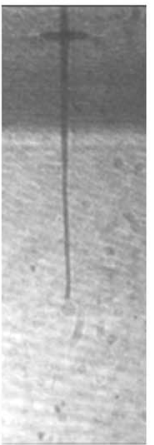

$4.1 \mu \mathrm{s}$

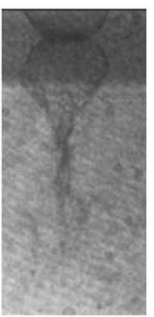

$1.6 \mu \mathrm{s}$

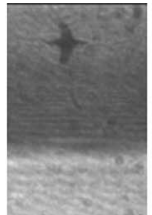

$30 \mu \mathrm{s}$

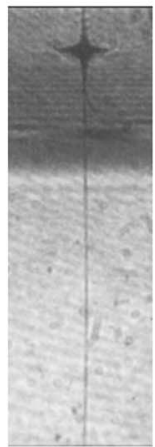

$90 \mu \mathrm{s}$

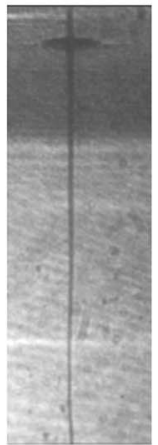

$7.6 \mu \mathrm{s}$

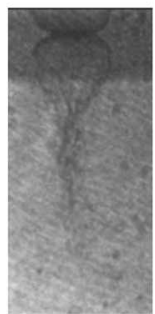

$1.8 \mu \mathrm{s}$

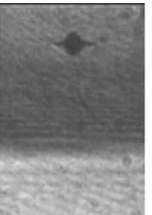

$75 \mu \mathrm{s}$

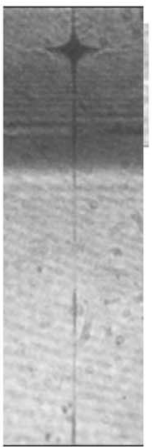

$100 \mu \mathrm{s}$

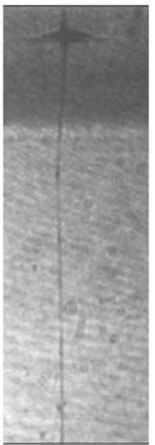

$50 \mu \mathrm{s}$

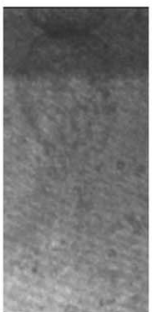

$2 \mu \mathrm{s}$

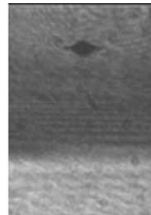

$100 \mu \mathrm{s}$

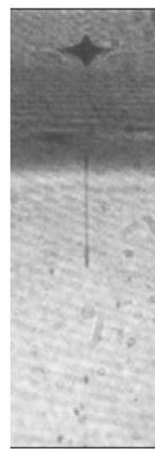

$120 \mu \mathrm{s}$

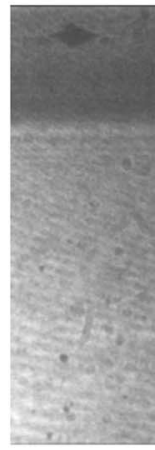

$100 \mu \mathrm{s}$

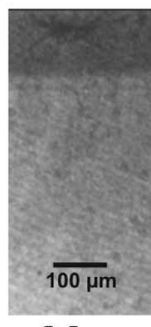

$2.2 \mu \mathrm{s}$

FIG. 3. Selected images of the time-resolved study of the LIFT process at different laser fluences: (a) 1.2, (b) 2.4, (c) 5.6, and (d) $10 \mathrm{~J} / \mathrm{cm}^{2}$.

The dynamics observed in this experiment at intermediate fluences, in which very long and stable jets develop, had never been reported by other research groups. Actually, in previous time-resolved LIFT experiments liquid ejection proceeded in a different way. ${ }^{21,22}$ Images of the LIFT process in the case of a barium-zirconium titanate/terpineol $\left(\mathrm{BaTiO}_{3}\right)$ suspension, ${ }^{21}$ a viscous paste with a rheology clearly different from that of the present experiment, showed the development of a turbulent and irregular jet which fragmented into multiple droplets short after its formation. On the other hand, and also under different conditions from those of the present experiment, images of the process using a bovine serum albumin solution ${ }^{22}$ showed the formation of a thin and blurred jet which broke into multiple droplets after traveling only $200 \mu \mathrm{m}$. Moreover, it also has to be noted that in the present experiment a direct correlation between the deposits and time-resolved images can be established. In fact, it has been found that for the range of laser fluences leading to the deposition of circular droplets liquid transfer is mediated by the formation of very long and stable jets, and therefore droplet deposition must be due to the contact of the long and stable jet with the receptor substrate, as we had previously pointed out. ${ }^{23}$

The analysis of the front position versus time for the four series of images reflects the already described characteristics of the respective dynamics (Fig. 4). The initial bubbleexpansion process can be easily identified in the four plots, showing a good alignment of the experimental points. The front velocity in this stage notably increases with laser fluence: $25 \mathrm{~m} / \mathrm{s}$ at $1.2 \mathrm{~J} / \mathrm{cm}^{2} ; 90 \mathrm{~m} / \mathrm{s}$ at $2.4 \mathrm{~J} / \mathrm{cm}^{2} ; 150 \mathrm{~m} / \mathrm{s}$ at 


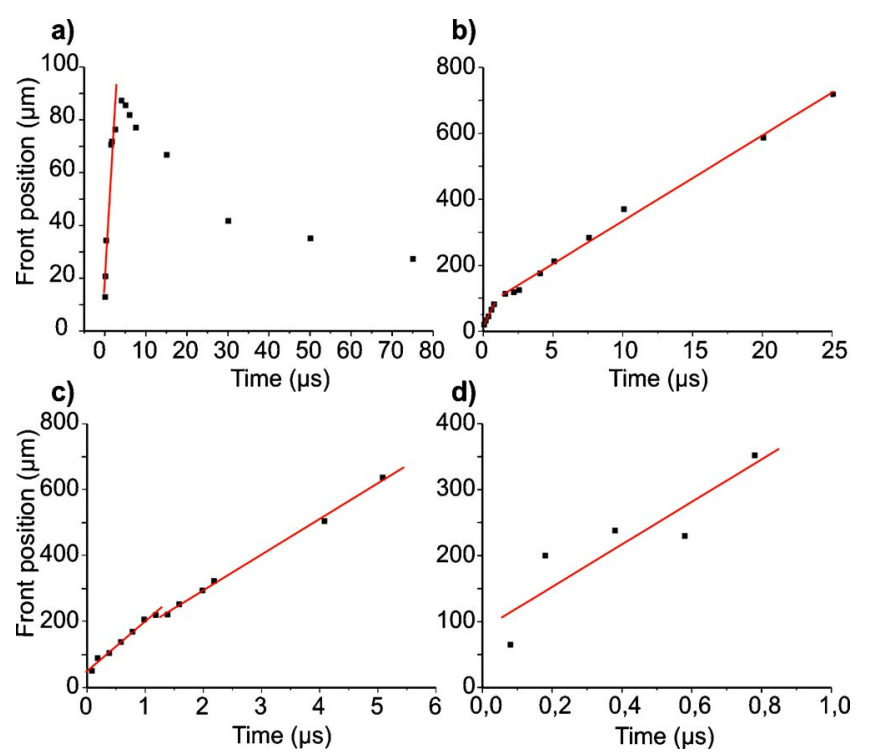

FIG. 4. (Color online) Plot of the liquid front position vs time at different laser fluences: (a) 1.2, (b) 2.4, (c) 5.6 , and (d) $10 \mathrm{~J} / \mathrm{cm}^{2}$.

$5.6 \mathrm{~J} / \mathrm{cm}^{2}$; and finally, $300 \mathrm{~m} / \mathrm{s}$ at $10 \mathrm{~J} / \mathrm{cm}^{2}$. At the lowest fluence [Fig. 4(a)], when bubble collapse and jet formation start, the front position slows down until the jet is fully developed. Then, the jet front undergoes the slow recoiling process described above which corresponds to a quasiexponential decay. At higher fluences, once the jet is fully developed it goes on advancing at constant velocity (25 and 110 $\mathrm{m} / \mathrm{s}$, respectively) until it disappears from the field of view of the camera. This velocity is always lower than that of the corresponding initial bubble expansion. Finally, at the highest fluences, since bubble bursting occurs, it is not possible to evaluate the front position after $780 \mathrm{~ns}$.

Time-resolved images of the process from the top view are presented in Fig. 5(a). The fluence used in this case was $3 \mathrm{~J} / \mathrm{cm}^{2}$. At these conditions, well-defined droplets are obtained, as it is proven by the presence of droplets in the background of the four frames. Moreover, all images show a dark circle, with a lighter central part and a halo. This circle corresponds to the vapor bubble described in the side view images. Actually, both the behavior of the bubble, with a diameter increasing for times up to $580 \mathrm{~ns}$ and stopping after this time, as well as the maximum dimensions of this diameter, around $100 \mu \mathrm{m}$, are consistent with the side view images obtained at a similar fluence [Fig. 3(b)]. The top view images also demonstrate that the bubble presents revolution

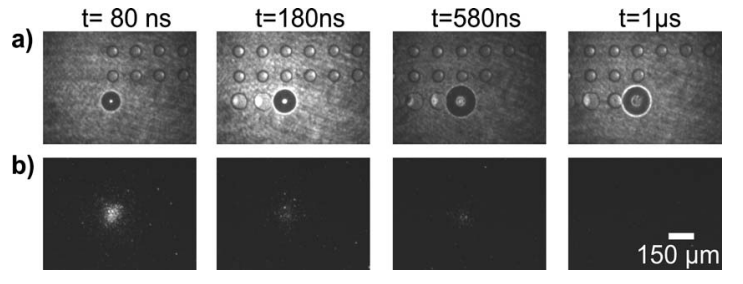

FIG. 5. Top view images of the transfer process using (a) an illuminating source, which allows visualizing the vapor bubble generated and (b) no illuminating source, which allows detecting the presence of a plasma. The bright points in the background of this last series are due to the high intensification of the MCP, which produces a low signal-to-noise ratio. symmetry around the axis of the laser beam. Therefore, it is expected that all the bubbles and jets observed in the side view images of the process (Fig. 3) also have such revolution symmetry. A second series of images was acquired at the same conditions as the first one, but without any illumination source [Fig. 5(b)]. In this case, the MCP was intensified at its maximum in order to detect any signal. It can be observed that a spark of light is initially generated, which progressively fades away until it completely disappears at $1 \mu \mathrm{s}$. This spark is an evidence of plasma formation. On the other hand, the presence of spots on the Ti film (not shown) at all analyzed fluences, even at those not leading to droplet deposition, confirms the ablation of the absorbing coating, which indicates that the generated plasma is due to the ablation of the Ti film.

\section{DISCUSSION}

All the described dynamics have in common the creation of a high-pressure vapor bubble which expands. The origin of such bubble can be attributed to the plasma formation found above. In fact, the expansion of a plasma inside a liquid, as it occurs in this experiment, is a well-known process which leads to the generation of a cavitation bubble. ${ }^{24-26}$ Therefore, the formation of the cavitation bubble in this LIFT experiment is induced by the plasma resulting from the ablation of the Ti film.

The dynamics of a cavitation bubble generated into a liquid and near its free surface has been previously studied. $^{27,28}$ Actually, if the cavitation bubble is generated close enough to the free surface, the bubble expansion can result in the formation of needlelike jets. ${ }^{27-31}$ Such situation is similar to that of the present LIFT experiment, although it is not exactly the same: the cavitation bubble generated in LIFT is not a freestanding bubble, contrary to those obtained in the reported cavitation experiments, due to the presence of the donor support; in addition, the aspect-ratio of the jets generated in LIFT is much higher than that of the reported jets. Despite these differences, those cavitation experiments can help describing, at least qualitatively, the dynamics of the LIFT process, as it is presented next.

The initial expansion of the LIFT generated bubble is due to a force balance: while the force exerted by the bubble internal pressure surpasses that due to the outward pressure plus the surface tension force, the expansion proceeds. However, such expansion is not isotropic: due to the relative ease with which the bubble displaces the liquid-air interface (free surface), which contrasts with the difficulty in displacing the surrounding liquid, the pole of the bubble becomes elongated, ${ }^{28}$ as it is observed in the first frames of the four series of Fig. 3. Theoretical calculations of cavitation bubbles experiments indicate that once the free surface is raised due to the bubble expansion process, flows along the liquid sheet displaced by the vapor and coming from the unperturbed liquid, meet in the pole, generating there a high pressure region. ${ }^{28}$ The process has been illustrated in the first two frames of Fig. 6. Following bubble expansion, three different situations have been observed to occur depending on the laser fluence: bubble collapse and formation of a jet, 


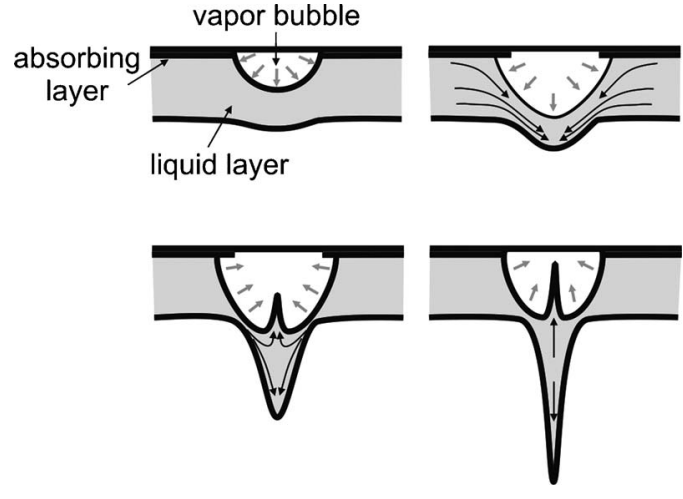

FIG. 6. Scheme of the bubble evolution and jet formation (from left to right and from up to down). The black arrows indicate liquid flow, and the gray arrows vapor bubble expansion and recoil.

which recoils [Fig. 3(a)], bubble collapse and formation of a progressive jet [Figs. 3(b) and 3(c)], and bubble bursting [Fig. 3(d)].

The bubble collapse and posterior jet formation for the first two cases has been schematically represented in the last two frames of Fig. 6. Since the bubble internal pressure decreases as the bubble expands, ${ }^{29}$ there exists a moment from which the balance between internal and external forces is favorable to the last ones, after which the bubble collapse initiates. This is a typical situation which leads to the development of needlelike jets. ${ }^{30}$ Actually, two needlelike jets flowing in opposite directions are usually produced: ${ }^{30,31}$ one away the free surface, as it is observed in Fig. 3, and another within the bubble (called counterjet), which cannot be appreciated in the time-resolved images (but illustrated in Fig. 6). The formation of such jets can be understood considering the fluid flows toward the symmetry axis depicted in Fig. 6. As stated before, they meet in the pole of the liquid sheet, where they generate a pressure maximum which forces the fluid toward those regions in which the pressure is lower: the free surface (jet) and the inside of the bubble (counterjet). ${ }^{31}$

At low laser fluences, once the jet is formed, the force exerted by the high pressure region generated between the bubble and the free surface is not high enough to overcome the surface tension force. Then, the jet recoils to its initial position [Fig. 3(a)]. In this case, since the maximum jet length is shorter than the donor film-receptor substrate distance, the jet cannot reach the receptor substrate and thus, no material is deposited. However, the reduction in this distance could lead to material deposition, as has already been experimentally demonstrated using a different solution. ${ }^{20}$

At higher laser fluences, in the intermediate fluence range, the jet inertia is high enough to surpass the recoiling force exerted by the surface tension. This allows the jet to maintain its progression, while becoming thinner as it advances [Figs. 3(b) and 3(c)]. As it has been pointed out above, such dynamics has also been observed in cavitation experiments near a free surface. ${ }^{27}$ Actually, calculations using the boundary integral method to solve the motion of a cavitation bubble near a free surface $^{28}$ have evidenced that the evolution of the free-surface position above the bubble is very similar to that of the present experiment [Figs. 4(b) and 4(c)]. Those calculations show that, initially, the front posi- tion of the raised free-surface advances at a high speed due to bubble expansion, but then it slows down due to bubble collapse, although it continues advancing in the form of a jet. Such jet advances at a constant speed, slower than that of the initial expansion process. These two different behaviors, one concerning bubble expansion and the other concerning jet advance at constant speed, are in good agreement with the results of the present experiment [Figs. 4(b) and 4(c)]. Moreover, the evolution with fluence of the results presented in Figs. 4(b) and 4(c) is well described by those calculations. At a fixed depth, as the maximum radius of the bubble increases, calculations show that the initial front speed increases, and the posterior speed reduction (when the jet develops) is less significant. In the present experiments, we observe the same evolution with the fluence increase: at $2.4 \mathrm{~J} / \mathrm{cm}^{2}$ the initial expansion speed is $90 \mathrm{~m} / \mathrm{s}$, which is reduced to $25 \mathrm{~m} / \mathrm{s}$ after jet development; on the other hand, at $5.6 \mathrm{~J} / \mathrm{cm}^{2}$, the initial speed is $150 \mathrm{~m} / \mathrm{s}$, much higher than in the previous case, and the speed after jet formation is 110 $\mathrm{m} / \mathrm{s}$, a much less significant reduction than before. An increase in fluence corresponds to an increase in the maximum radius that the bubble attains during its expansion (Fig. 3), which indicates that the behavior predicted in Ref. 28 also describes well the evolution in fluence found in the experiments.

The jet advance does not last forever, but when the jet has reached a long length it becomes unstable and finally breaks due to surface tension effects in the so called PlateauRayleigh instability, as is common in thin liquid streams. ${ }^{32}$ The maximum jet length in the present experiments cannot be directly measured, since the jet front surpasses the field of view of the camera before breakup. However, assuming that the jet advances at constant speed until breakup, its maximum length can be estimated to be of the order of some millimeters.

A very different dynamics occurs at the highest laser fluences. In this case, instead of bubble collapse and jet formation, the bubble bursts. This is due to the high bubble pressure resulting from such energetic laser pulses, which can exert a force on the liquid surface strong enough to overcome the force due to surface tension, leading to the burst of the liquid sheet surrounding the bubble. ${ }^{28}$ Such dynamics can be related to the deposits of the upper rows in Fig. 1 which correspond to these fluences: when the laser fluence surpasses a certain threshold, bubble bursting is the responsible for the splashing obtained on the receptor substrate. Actually, the deposited material at high fluences (Fig. 1) presents a circular halo, with a diameter comparable to the lateral dimensions of the vapor bubble observed in the initial frames of Fig. 3(d). This suggests that the bubble initially reaches the receptor leaving a circular mark (the halo), and afterwards it bursts producing splashing. Such behavior clearly indicates that the surface tension of the liquid solution is a key parameter which sets the fluence resulting in splashing. Moreover, since the recoiling of the jet is also due to surface tension effects, it can be concluded that this parameter determines the range of fluences resulting in regular and well defined droplet deposition: a low surface tension would result in bubble bursting at low energies and consequent 
splashing on the receptor, whereas a high surface tension would require high energies to avoid the recoiling of the jet front and thus produce material deposition.

\section{CONCLUSION}

The preparation of a microarray through LIFT at different laser fluences has evidenced the existence of a fluence range in which regular and well-defined droplets are deposited. At lower fluences no material is deposited, and at higher fluences, irregular droplets and splashing are obtained.

High-speed images of the transfer process dynamics from the side view have shown that initially, and at all the analyzed fluences, a vapor bubble is generated. The origin of such bubble has been found to be, thanks to time-resolved imaging of the process from the top view, the plasma formed after laser ablation of the absorbing layer. Once the vapor bubble is formed, it suffers a high-speed expansion process, which can evolve into three different situations, depending on the laser fluence.

At low fluences, after the expansion process, the bubble collapses and a needlelike jet is developed due to the high pressure region generated in the bubble pole. Such high pressure is created by the liquid flows toward the bubble pole during bubble expansion. However, the jet is not energetic enough to advance overcoming surface tension forces, and it starts a recoiling process until it finally disappears. Consequently, if the liquid-receptor separation is higher than the jet length, the jet does not contact the receptor and thus no material is deposited on it.

At intermediate fluences, the initial bubble expansion and posterior jet formation is analogous to the previous situation. However, in this case the jet has enough inertia to continue advancing at constant velocity while progressively thinning. Such dynamics is very similar to that observed in cavitation bubbles generated close to a free surface, and theoretical calculations of the expansion of such a cavitation bubble predict a behavior which is in good agreement with that observed in the present results. Finally, when the jet has reached a very long length, it becomes unstable due to surface tension effects and breakup occurs. At these conditions, deposition of well-defined circular droplets is achieved.

At high fluences, the bubble expansion is so violent that it overcomes surface tension resulting in bubble bursting. This situation generates splashing on the receptor substrate.

\section{ACKNOWLEDGMENTS}

This work is part of a research program funded by MCI of the Spanish Government (Project Nos. MAT2007-62357 and CSD2008-00023), Fondo Europeo de Desarrollo Regional (FEDER), and DIUE of the Catalan Government. Professor Alfred Vogel from the University of Lübeck is acknowledged for useful discussions about jetting mechanisms.

${ }^{1}$ Y.L. Loo and I. McCulloch, MRS Bull. 33, 653 (2008).

${ }^{2}$ T. Boland, T. Xu, B. Damon, and X. Cui, J. Biotechnol. 1, 910 (2006).

${ }^{3}$ D. Janasek, J. Franzke, and A. Manz, Nature (London) 442, 374 (2006).

${ }^{4}$ P. Calvert, Chem. Mater. 13, 3299 (2001).

${ }^{5}$ H. Sirringhaus and T. Shimoda, MRS Bull. 28, 802 (2003).

${ }^{6}$ C.B. Arnold, P. Serra, and A. Piqué, MRS Bull. 32, 23 (2007).

${ }^{7}$ P. K. Wu, B. R. Ringeisen, D. B. Krizman, C. G. Frondoza, M. Brooks, D. M. Bubb, R. C. Y. Auyeung, A. Piqué, B. Spargo, R. A. McGill, and D. B. Chrisey, Rev. Sci. Instrum. 74, 2546 (2003)

${ }^{8}$ J. M. Fernández-Pradas, M. Colina, P. Serra, J. Domínguez, and J. L. Morenza, Thin Solid Films 453-454, 27 (2004).

${ }^{9}$ M. Colina, M. Duocastella, J. M. Fernández-Pradas, P. Serra, and J. L. Morenza, J. Appl. Phys. 99, 084909 (2006).

${ }^{10}$ V. Dinca, M. Farsari, D. Kafetzopoulos, A. Popescu, M. Dinescu, and C. Fotakis, Thin Solid Films 516, 6504 (2008).

${ }^{11}$ H. Kim, C. Y. Auyeung, and A. Piqué, J. Power Sources 165, 413 (2007).

${ }^{12}$ C. Boutopoulos, V. Tsouti, D. Goustouridis, S. Chatzandroulis, and I. Zergioti, Appl. Phys. Lett. 93, 191109 (2008).

${ }^{13}$ B. Hopp, T. Smausz, Z. Antal, N. Kresz, Z. Bor, and D. Chrisey, J. Appl. Phys. 96, 3478 (2004).

${ }^{14}$ N. T. Kattamis, P. E. Purnick, R. Weiss, and C. B. Arnold, Appl. Phys. Lett. 91, 171120 (2007).

${ }^{15}$ M. Duocastella, J. M. Fernández-Pradas, P. Serra, and J. L. Morenza, Appl. Phys. A: Mater. Sci. Process. 93, 941 (2008).

${ }^{16}$ H. Kim, G. P. Kushto, C. B. Arnold, Z. H. Kafafi, and A. Piqué, Appl. Phys. Lett. 85, 464 (2004).

${ }^{17}$ R. Fardel, M. Nagel, F. Nüesch, T. Lippert, and A. Wokaun, Appl. Phys. Lett. 91, 061103 (2007).

${ }^{18}$ P. Serra, M. Colina, J. M. Fernández-Pradas, L. Sevilla, and J. L. Morenza, Appl. Phys. Lett. 85, 1639 (2004).

${ }^{19}$ M. Colina, P. Serra, J. M. Fernández-Pradas, L. Sevilla, and J. L. Morenza, Biosens. Bioelectron. 20, 1638 (2005).

${ }^{20}$ M. Duocastella, M. Colina, J. M. Fernández-Pradas, P. Serra, and J. L. Morenza, Appl. Surf. Sci. 253, 7855 (2007).

${ }^{21}$ D. Young, R. C. Y. Auyeung, A. Piqué, D. B. Chrisey, and D. D. Dlott, Appl. Surf. Sci. 197-198, 181 (2002).

${ }^{22}$ J. A. Barron, H. D. Young, D. D. Dlott, M. M. Darfler, D. B. Krizman, and B. R. Ringeisen, Proteomics 5, 4138 (2005).

${ }^{23}$ M. Duocastella, J. M. Fernández-Pradas, P. Serra, and J. L. Morenza, Appl. Phys. A: Mater. Sci. Process. 93, 453 (2008).

${ }^{24}$ C. B. Schaffer, N. Nishimura, E. N. Glezer, A. M. T. Kim, and E. Mazur, Opt. Express 10, 196 (2002).

${ }^{25}$ A. Vogel, S. Busch, and U. Parlitz, J. Acoust. Soc. Am. 100, 148 (1996).

${ }^{26}$ E. A. Brujan and A. Vogel, J. Fluid Mech. 558, 281 (2006).

${ }^{27}$ J. R. Blake and D. C. Gibson, J. Fluid Mech. 111, 123 (1981).

${ }^{28}$ A. Pearson, E. Cox, J. R. Blake, and S. R. Otto, Eng. Anal. Boundary Elem. 28, 295 (2004).

${ }^{29}$ C. E. Brennen, Cavitation and Bubble Dynamics (Oxford University Press, New York, 1995), p. 79.

${ }^{30}$ E. A. Brujan, K. Nahen, P. Schmidt, and A. Vogel, J. Fluid Mech. 433, 251 (2001).

${ }^{31}$ J. P. Frank and J. M. Michel, Fundamentals of Cavitation (Kluwer, Dordrecht, 2004), p. 57.

${ }^{32}$ J. Eggers, Rev. Mod. Phys. 69, 865 (1997). 